\title{
HARDY'S TYPE INEQUALITY FOR THE OVER CRITICAL EXPONENT ASSOCIATED WITH THE DUNKL TRANSFORM
}

\author{
RAHMOUNi ATEF
}

Abstract. For the Hardy space $H_{q, s}^{p}\left(\mathbb{R}^{d}, \mu_{k}\right), 0<p \leqslant 1$, we shall improve a Hardy's type inequality associated with the Dunkl transform respect to the measures $\mu_{\kappa}$ homogeneous of degree $\gamma$, on the strip $(2 \gamma+d)(2-p) \leqslant \sigma<2 \gamma+d+p(N+1)$, where $N=[(2 \gamma+d)(1 / p-1)]$ is the greatest integer not exceeding $(2 \gamma+d)(1 / p-1)$.

Mathematics subject classification (2010): 42B10, 42B30, 33C45.

Keywords and phrases: Hardy space, Hardy's type inequality, Dunkl transform.

\section{REFERENCES}

[1] M. Assal, Hardy's type inequality associated with the Hankel transform for over critical exponent, Integr. Transf. Spec. F., 22 (2011), 45-50.

[2] M. Assal And A. Rahmouni, Hardy's type inequality associated with the Laguerre Fourier transform, Integr. Transf. Spec. F., 24 (2013), 156-163.

[3] M. Assal And A. Rahmouni, An improved Hardy's inequality associated with the Laguerre Fourier transform, Collect. Math., (2013), 1-11.

[4] R. R. Coifman, A real-variable characterization of $H^{p}$, Studia Math., 51 (1974), 269-274.

[5] M. F. E. DE JEU, The Dunkl transform, Invent. Math., 113 (1993), 147-162.

[6] C. F. DunkL, Differential-difference operators associated to reflection groups, Trans. Amer. Math. Soc., 311 (1989), 167-183.

[7] C. F. DunKL, Integral kernels with reflection group invariance, Canad. J. Math., 43 (1991), 1213 1227.

[8] C. F. DunKL, Hankel transforms associated to finite reflection groups, Contemp. Math., 138 (1992), $123-138$.

[9] C. FefFerman and E. M. Stein, $H^{p}$ spaces of several variables, Acta Math., 129, (1972), $137-$ 193.

[10] G. B. Folland And E. M. Stein, Hardy spaces on homogeneous groups, Princeton University Press, Princeton, NJ, 1982.

[11] J. Garcia-Cuerva And J. Rubio de Francia, Weighted norm inequalities and related topics, North Holland, 1985.

[12] J. E. HumphreYs, Reflection groups and coxter groups, Cambridge Univ. Press, 1990, 1-204.

[13] H. MeJjaOli, Littlewood-Paley decomposition associated with the Dunkl operators and paraproduct operators, J. Inequal. Pure and Appl. Math., 9 (4) (2008), 1-25.

[14] A. Rahmouni And M. Assal, An improved Hardy's inequality associated with the Euclidean Fourier transform, Acta Mathematica Scientia, 33 B (5) (2013), 1382-1386.

[15] A. Rahmouni AND M. Ass AL, Hardy's type inequality for the critical exponent associated with the inverse Laguerre Fourier transform, Integr. Transf. Spec. F., (2013) 1-9.

[16] A. Rahmouni, M. Assal And M. N. Lazhari, An improved Hardy's inequality associated with the dual of Laguerre hypergroup, (preprint).

[17] M. RöSLER, Positivity of Dunkl's intertwining operator, Duke Math. J., 98 (1999), 445-463.

[18] M. RöSLER, A positive radial product formula for the Dunkl kernel, Trans. Amer. Math. Soc., 355 (2003), 2413-2438. 
[19] E. M. STEIn, Harmonic Analysis, real variable methods, orthogonality and oscillatory integrals, Princeton Univ. Press, Princeton, NJ, 1993.

[20] F. Soltani, Paley type inequality on the Hardy type space in the Dunkl setting, Arch. Math., 95 (2010), 35-44.

[21] M. H. TAIBleson AND G. Weiss, The molecular characterization of certain Hardy spaces, Astérisque 77 (1980), Société Math. de France, Paris, 67-149.

[22] S. Thangavelu And Y. XU, Convolution operator and maximal function for the Dunkl transform, J. Anal. Math., 97 (2005), 25-56.

[23] K. TRIMÈCHE, The Dunkl intertwining operator on spaces of functions and distributions and integral representation of its dual, Integr. Transf. and Special Funct., 12 (2001), 349-374. 